\title{
La perspectiva de enfermos crónicos sobre la atención médica en Guadalajara, México. Un estudio cualitativo
}

\author{
Perspectives of chronically ill patients \\ concerning medical care in Guadalajara, \\ Mexico. A qualitative study
}

Francisco J. Mercado Martínez 1

I gor M. Ramos Herrera 2

Enriqueta Valdez Curiel 2

\footnotetext{
1 Departamento de Salud Pública, Centro Universitário de Ciências de la Salud, Universidad de Guadalajara. Apartado Postal 1-2044, Guadalajara, México. fmercado@cucs.udg.mx 2 Departamento de Salud Pública, Centro Universitário de Ciências de la Salud. Sierra Mojada 950, Col. Independencia, Guadalajara 44340, México.
}

Abstract This paper reports partial findings from a broader study on the experience of people with chronic diseases. The objective was to explore the perspectives of diabetic patients towards medical care. A qualitative study was conducted in a poor neighborhood of Guadalajara, Mexico. Thirty subjects with diabetes mellitus participated in the study. Data was gathered by open and semi-open interviews in the subjects' homes and over the course of one year. Data were analyzed using a combination of content and conversational analyzing techniques. Three perspectives predominated when participants evaluated medical care: some define it as good, some as ambivalent, and the rest as bad. These perspectives were cl osely linked to their disease experience and available medical opti ons according to their material resources. These perspectives change with time, are specific to each available service and type of medical care, and are constructed in terms of all the subjects' present chronic illnesses. Those treated through the social security system evaluate the care in negative terms, with the opposite occurring with those treated in public health care centers and private facilities. I mplications regarding health care reform are discussed. Key words Patients; Chronic Diseases; Health Services

Resumen Este trabajo reporta los hallazgos parciales de un estudio más amplio sobre la experiencia de vivir con enfermedades crónicas; el mismo se centra en la perspectiva de suj etos enfermos sobre los servicios de salud. Un estudio cualitativo fue realizado en un barrio popular de Guadalajara, con treinta suj etos con diabetes. La información se obtuvo mediante entrevistas semi y no estructuradas apl icadas en sus hogares. Los datos se obtuvieron durante un año, los mismos fueron analizados mediante análisis de conteni do y conversacional. Tres valoraciones generales identificamos sobrela atención médica por parte de los partici pantes del estudio: algunos la consi deran buena, para otros es ambival ente y para el resto es mala. Estas valoraciones se reIacionan estrechamente con la trayectoria del padecimiento y los recursos materiales disponibl es que posibilitan optar por distintos tipos de servicios. Tales perspectivas son cambiantes a lo largo del tiempo, incluyen los diversos servi ci os existentes y se construyen incorporando otros padecimientos crónicos. Quienes acuden al Seguro Social la definen en términos eminentemente negativos, a diferencia de aquellos con acceso a los servicios públicos de asistencia y a la medicina privada. Se discuten sus implicaciones en referencia a la reforma del sector sal ud.

Palabras clave Enfermos; Enfermedad Crónica; Servicios Médicos 


\section{Introducción}

Desde hace décadas, se reconoce ampliamente la necesidad de tomar en consideración e incorporar el punto de vista de la población en la planeación, organización y evaluación de los servicios de salud. La creciente tendencia a considerar estos servicios como empresas y a los usuarios como consumidores ha incrementado el interés de gobiernos e instituciones por conocer la opinión de estos últimos con respecto a la atención que reciben y la satisfacción de sus expectativas (Aguirre, 1990; WHO, 1990).

A inicios del nuevo milenio poco se ha avanzado en el conocimiento de la perspectiva de la comunidad, de usuarios, enfermos o clientes sobre los servicios de salud en numerosos países, incluyendo M éxico. El asunto de ninguna forma es irrelevante. Frente a aquellas propuestas de mejorar los servicios médicos en forma gradual, un número creciente de autores subraya la necesi dad de introducir cambios radicales en los mismos, destacando la cada vez más raquítica inversión en el sector, el porcentaje de la población sin acceso a ellos, la multiplicidad de servicios de salud existentes a la par de la desigualdad en la asignación de los recursos a los sectores sociales. En fechas recientes, también aparece en la agenda de discusión de partidos políticos, organizaciones sociales y académicos un rechazo a las propuestas de privatización de los servicios de salud, sobre todo de la seguridad social. Sin embargo, frente a la diversidad de diagnósticos y propuestas de los actores involucrados en el asunto (Buller \& Buller, 1987; Aguirre, 1990; Ruiz et al., 1990; Ramírez-Sánchez et al., 1998), todos justificando sus argumentos en el bien de la población, resulta paradójica la ausencia de información que ofrezca una visión sistemática de las propuestas de la misma población sobre dichos servicios.

La realización de estudios de esta naturaleza en sujetos con enfermedades crónicas adquiere relevancia en tanto involucran a un porcentaje elevado de la población; es un sector con requerimientos constantes de atención médica y durante largos periodos de su vida. De esta manera, la cronicidad de su padecimiento los convierte en los receptores más frecuentes de los servicios de salud disponibles, cualquiera sea su tipo y calidad. Por ende, se asume que a ellos les correspondería, también, dar la última palabra sobre el rumbo de dichos servicios para garantizar la atención necesaria.

Este trabajo reporta los hallazgos parciales de un estudio más amplio encaminado a conocer la experiencia de vivir con una enfermedad crónica. Aquí presentamos la perspectiva de sujetos enfermos sobre la atención médica e intentamos responder a varias preguntas: ¿cuál es el punto de vista de los sujetos respecto a la atención médica de su padecimiento?, ¿difiere la perspectiva de quienes acuden a la seguridad social respecto a la de quienes acuden a la asistencia pública?, ¿cuán favorable es la perspectiva de los enfermos sobre la atención médica que reciben?, en caso de ser negativa, ¿qué aspectos o tópicos aparecen con mayor recurrencia?, ¿existen diferencias sociodemográficas o del mismo padecimiento entre quienes adoptan posturas distintas?

\section{Estado del arte}

En años recientes, se han realizado numerosos estudios para conocer el punto de vista de los usuarios y de la población sobre la atención médica, tanto en países centrales como en periféricos (Buller \& Buller, 1987; Conrad, 1987; Lewis, 1994; Pinheiro \& Travassos, 1999). La mayoría, aunque emplean diversas posiciones teóricas, evalúan los servicios de salud desde la perspectiva de los profesionales de la salud. Muchos autores, incluso, parecen contribuir a perpetuar el status quo de una atención médica deficiente y deshumanizada, motivo por el cual han pasado a ser objeto de no pocas críticas. El uso de cuestionarios con respuestas cerradas, por ejemplo, limita la libre expresión de quienes viven el padecimiento y coarta de manera sutil el proceso reflexivo del sujeto estudiado. El lenguaje, el orden y el enfoque empleados tradicionalmente en estas investigaciones representan un modo de expresión ajeno a los sujetos acostumbrados al diálogo abierto, o al silencio. Dejar de lado el contexto de los actores sociales, como ocurre con muchos estudios, no posibilita entender los aspectos particulares de cada enfermedad y el efecto que el entorno socioeconómico ejerce sobre el individuo, limitando así la generación de información pertinente para proponer los cambios necesarios (Aguirre, 1990; Ruiz et al., 1990). Lara et al. (in press) mencionan que el estudio de las necesidades de salud y la utilización de los servicios requieren del uso combinado de aproximaciones cuantitativas y cualitativas. Esto permitiría ir más allá de la descripción epidemiológica o de la asociación estadística de variables. El uso exclusivo de los modelos cuantitativos puede eliminar las características individuales del uso de los servicios ya que se enfocan exclusivamente en la evaluación de los programas y operacionalizan erróneamente 
los términos al tratarlos como variables cuantitativas.

Frente a tal panorama, se viene consolidando una corriente bajo una orientación etnográfica y/ o cualitativa centrada en el punto de vista de los actores sociales sobre la atención a la enfermedad (Linder-Pelz, 1982; Modena, 1990; Huby, 1997). Esta corriente, cuyo surgimiento se ubica en las ciencias sociales, rescata la descripción de los enfermos sobre su experiencia con la enfermedad, su interpretación sobre la misma y la atención a su padecimiento, así como también la concepción que el sujeto tiene sobre su salud y su lugar en la sociedad (Herzlich \& Pierret, 1988). En el caso latinoamericano, este es un campo explorado hasta fechas recientes (Castro, 1995; Gattinara et al., 1995; Santos, 1995; Rocha et al., 1997; Mercado, 1998; Mercado et al., 1999; Mercado \& Torres, 2000), pero no por ello carente de importancia.

El incremento de los padecimientos crónicos y los cambios inherentes al campo de la salud, entre otros factores, han Ilevado a revalorar el papel de diversos actores sociales en torno al proceso de atención a la enfermedad. Y son los sujetos que viven con una enfermedad quizá el componente más importante del sistema, pero también el más ignorado. En el caso de México, los programas y las políticas de salud han sido planeados y ejecutados sin tomar en consideración su voz y sus propuestas que suelen ser considerados como receptores pasivos de dichos programas (Mercado, 1996). Al justificar su exclusión, varios autores argumentan la falta de capacidad de los mismos para hacer juicios sobre los aspectos que intervienen en la calidad de los servicios de salud, especialmente los técnicos (Like \& Zyzanski, 1987). Para otros, en cambio, los sujetos enfermos tienen la capacidad de discriminar todos los aspectos de la atención médica, razón por la cual aceptan y validan los resultados que apuntan en esa dirección (Jung et al., 1998).

La orientación cualitativa ha mostrado ser una opción para explorar la experiencia, las representaciones y las estructuras de significación en torno a los servicios de salud y las prácticas de atención a la enfermedad. A través de ella los actores sociales se expresan en sus términos, proyectan una imagen de sí mismos, de como se relacionan con el mundo y como les gustaría ser vistos y tratados por los demás. Esta corriente cualitativa permite, además, contextualizar el medio donde los sujetos construyen sus conceptos y experiencias, y también identifica la posición de quien habla y como se ve como enfermo. Permite, además, valorar no sólo la atención médica ofrecida, sino recono- cer al sujeto que sufre y los factores que intervienen en la forma como percibe tal atención en el contexto de su vida diaria (Hopton et al., 1993). Cuando la perspectiva de quien vive la enfermedad es considerada para planificar y ejecutar la atención médica, no sólo se incrementan las posibilidades de mejorar la calidad de los servicios, sino también el cumplimiento del tratamiento y el auto-cuidado.

\section{El panorama de los servicios de salud}

El sistema de atención médica en México se caracteriza, entre otras cosas, por la multiplicidad de instituciones existentes, su acceso diferencial, la disponibilidad limitada de recursos financieros y la inequidad en su distribución y asignación.

Múltiples instituciones conforman los servicios de salud. Sus orígenes y organización responden en lo fundamental a la forma como la población se inserta en la vida productiva. Menos del $10 \%$ del total, correspondiente a las clases altas y un sector de la media, tienen acceso permanente a la medicina privada; poco menos de la mitad son derechohabientes de la seguridad social; una cifra similar, correspondiente a sectores desfavorecidos de la sociedad, tiene acceso a los servicios públicos asistenciales, a través de la Secretaría de Salud. Pero otra cifra menor al $10 \%$, compuesta por el sector social más pobre, no tiene acceso a servicio médico alguno (Poder Ejecutivo Federal, 1995).

Otra característica del sector salud mexicano es la escasez permanente de recursos de que dispone. Tomando en consideración sólo tres rubros, es posible constatar que, desde inicios de la década de los ochentas, ha habido un estancamiento, y frecuentemente retrocesos, en los gastos per cápita en salud, el porcentaje del gasto en salud respecto del gasto público total y el porcentaje del gasto en salud respecto del producto nacional bruto. Referente al gasto personal en salud, por poner sólo un caso, hubo una disminución del 38\% entre 1981 y 1989 (Laurell, 1991).

Pero dicho sistema médico también se caracteriza por su inequidad, tal como se evidencia al tomar en cuenta la asignación de los recursos. Las instituciones de seguridad social recibieron el $44 \%$ del presupuesto en salud del país entre 1992 y 1994, aquellas orientadas a la población no asegurada apenas el 13\% y al sector privado correspondió el $43 \%$ del total. Tal inequidad también se constata al comparar el gasto per cápita entre las instituciones. La inversión de dos instituciones de seguridad social es notoriamente superior a la de la Secretaría de 
Salud; a saber, Petróleos Mexicanos invirtió 1.708 dólares americanos en 1994, el Seguro Social, 628 dólares y la Secretaría de Salud, apenas 161 dólares. La inversión en dicha Secretaría, según estos datos, es cuatro veces inferior a la del Seguro Social y casi once veces menor a la dePetróleos Mexicanos (Hernández et al., 1997).

La estructura de los servicios públicos es relativamente semejante, aunque con distintos recursos en ambas. El primer nivel cuenta con centros o clínicas de salud, y la Secretaría de Salud con médicos generales, mientras que el Seguro con especialistas en medicina familiar.

\section{Aspectos metodológicos y operacionales}

Diversas propuestas existen para indagar la perspectiva de la población enferma sobre la atención médica. Aquí optamos por realizar un estudio cualitativo a fin de rescatar el punto de vista de quien vive con la enfermedad crónica. El trabajo de campo, realizado entre octubre de 1997 y septiembre de 1998, nos posibilitó conocer la atención médica desde la óptica de los sujetos (Mercado et al., 1999). La orientación teórica adoptada es la crítico-interpretativa; la misma se expone con mayor detenimiento en Mercado (1996).

Oblatos, barrio popular (Selby et al., 1990) de 30 mil habitantes y ubicado al oriente de Guadalajara, México, fue el área de realización del estudio. Su origen data de la década de los sesentas, cuenta con los servicios públicos, también dispone de varios consultorios médicos, pequeñas clínicas particulares y laboratorios de análisis clínicos y farmacias. Sin embargo, las unidades médicas de seguridad social y de los servicios públicos asistenciales se ubican en los barrios adyacentes. Los hospitales de ambas instituciones se hayan en otras zonas de la ciudad, tal cual los consultorios de los médicos especialistas privados.

De los 756 sujetos con diabetes, identificados previamente en el barrio, seleccionamos 30 en base al razonamiento del muestreo teórico (Rodríguez et al., 1996). Nuestra intención en ningún momento fue contar con una muestra representativa de la población general, ni aspirábamos a la generalización de los resultados. Tal estrategia de selección obedeció a la necesidad de indagar sobre ciertas preocupaciones teóricas en torno al tema en cuestión; ésto es, se aspiraba a incorporar población con acceso a distintos servicios médicos, con ocupaciones e ingresos diversos, y diferentes en cuanto a edad y género. Una vez seleccionadas las per- sonas, fueron visitadas en sus hogares para explicarles los objetivos y dinámica del estudio e invitarlas a participar.

Los sujetos participantes tienen entre $35 \mathrm{y}$ 72 años y más de la mitad son mujeres. Además de la diabetes, tres de cada cinco tienen otra enfermedad crónica, sobre todo hipertensión o artritis. Según los criterios empleados por González-de-la-Rocha (1986) para el caso de Guadalajara, todos pertenecen a la clase trabajadora urbana, aunque tienen distintas ocupaciones e ingresos: casi un tercio, todas amas de casa y cuyos cónyuges trabajan en el sector informal, no tienen ingresos propios; cuatro sujetos son propietarios de pequeños negocios; ocho más se dedican al comercio informal, como vendedores en mercados ambulantes; siete son jubilados y dos son asalariados.

Cuatro participantes fueron entrevistados una vez, quince dos veces, y los once restantes en tres ocasiones. El intervalo entre las entrevistas fue de cuatro a seis meses. Las entrevistas fueron de tipo semi y no estructuradas. Las entrevistas fueron grabadas y su duración fue de una a dos horas. Las grabaciones se transcribieron literalmente. A excepción de dos casos, dos integrantes del equipo, un hombre y una mujer, participamos en las entrevistas. La presencia de entrevistadores de ambos sexos, asumimos en aquel momento, facilitaría la comunicación e incrementaría la confianza con los participantes.

Los datos fueron examinados inicialmente mediante el análisis de contenido textual, de acuerdo a Weber (1990) y Minayo (1997). Luego se realizó un análisis conversacional con el fin de identificar el lenguaje empleado y la interacción en la cual surgió la información (Gumperz, 1995). También seleccionamos partes de las narraciones para ilustrar con mayor fuerza situaciones particulares a modo de citas textuales. De los temas identificados en el estudio general, uno fue la perspectiva sobre la atención médica y su valoración general. La atención fue catalogada como buena, ambivalente y mala en base al tipo y orientación de los comentarios, tanto generales como específicos, de los participantes sobre los servicios médicos recibidos en torno a su enfermedad crónica a lo largo del tiempo. El análisis detallado de la información nos mostró que las perspectivas variaban con el tiempo y ante los distintos servicios; por ello, en ciertos momentos se procedió a manejarla en conjunto, y en otros sólo se alude a la fuente primaria de atención.

En este trabajo, utilizamos el concepto trayectoria del padecimiento acorde a los planteamientos formulados por Mercado et al. (1998). 
Tal concepto lo entendemos como aquel fenómeno cambiante que comprende las vidas, las historias, las percepciones y las actitudes de los individuos en torno a su padecimiento; dicho en otras palabras, se trata de un concepto que traslada al centro de la discusión la dimensión social, emocional y cultural del padecimiento a lo largo del tiempo. Los nombres de los sujetos participantes fueron sustituidos por nombres ficticios con el fin de proteger su identidad.

\section{La atención médica desde la mirada de los enfermos}

Tres val oraciones generales sobre la atención médica destacan entre los participantes del estudio. Un poco más de la tercera parte la considera buena, para otros tantos es ambivalente y para los restantes es mala o deficiente. Los primeros suelen describirla en sus narraciones en términos favorables, aceptables o útiles a lo largo del tiempo; los últimos destacan primordialmente sus rasgos negativos o sus limitaciones permanentes. Los segundos Ia catalogan, sea en conjunto o en partes, de forma semejante a los primeros durante una entrevista, pero como mala, deficiente, no confiable e inútil en otra, o inclusive en un momento posterior de la misma entrevista. Tales alusiones remiten a un cúmulo de representaciones y prácticas, pero sobre todo a sus experiencias al estar en contacto con los servicios de atención a su(s) padecimiento(s).

\section{De las valoraciones y sus argumentos}

Estas valoraciones sobre la atención médica responden a un cúmulo de circunstancias entremezcladas. Los participantes con una valoración buena o favorable son hombres y mujeres con una trayectoria estable del padecimiento, ésto es, con mínimos o nulos trastornos y/o efectos negativos en su vida diaria, con menos años con diabetes (nueve en promedio), un menor número de padecimientos crónicos (1,6, en promedio) y quienes manifiestan menos necesidad de atención médica especializada, la cual logran en forma satisfactoria; en tanto varios disponen de recursos económicos suficientes para acceder a la medicina privada, mientras que los restantes acuden en ocasiones a los servicios públicos donde reciben medicamentos, realizan estudios clínicos y adquieren medicamentos en forma gratuita. Sólo dos personas tienen una opinión favorable de la seguridad social. Se trata de un hombre jubilado, quien no tiene molestias a causa de la diabetes, y no le preocupa el tiempo destinado a la consulta al no trabajar. La otra persona acude a una institución de seguridad diferente al Seguro y, al igual que una que asiste a la medicina privada sin tener suficientes recursos, su opinión es favorable porque cuenta con parientes que allí laboran y le facilitan la atención. Juan Núñez, de 60 años, con diabetes e hipertensión, siempre se manifestaba orgulloso de sus pocas molestias hasta el momento de presentar un infarto. A pesar de los trastornos recientes, siempre está satisfecho de la atención recibida en la seguridad social porque su esposa trabaja en el hospital donde es atendido. Una tarde, antes de presentar el infarto, nos decía "a los dos días quesalí (del hospital) ... me dijo mi mujer 'vamosa que te dé al go un doctor que trabaja en ... vamos para ... preguntarle con más confianza como vas'... ella tiene facilidad de que se los presten (Ios estudios), y entonces los Il evó así de contrabando y se los enseño ... (también) las laboratoristas son muy amigas de mi mujer ... es que no tenemos que sacar ficha, por eso voy hasta allá; allá no hay que'véngase a las si ete de la mañana', pos yo no podría, yo regreso detrabajar a las nueve... Y ella les habla 'oye, me haces el favor' y ya ... y ll lego y !pum! luego luego mesacan la sangre y al día siguiente, más bien ese mismo día, ya se trae el resultado ...".

Los participantes con una valoración ambivalente de la atención médica son aquellos en quienes se mezclan circunstancias diversas, aunque todos tienen acceso al Seguro Social. Son personas del sexo masculino y femenino, con trayectorias estables y oscilatorias. Aquellos con trayectorias estables son más jóvenes, sólo presentan diabetes y tienen menos años de padecerla; sin embargo, no cuentan con recursos para acceder a la medicina privada, por lo cual asisten ocasionalmente al Seguro Social. Los otros tienen una trayectoria oscilatoria, son de más edad, tienen dos o tres padecimientos y más años con diabetes, tampoco cuentan con recursos económicos adicionales para la atención en la medicina privada, por lo cual acuden al Seguro Social o a los servicios públicos. José Ortiz, a sus 63 años, tiene una posición ambivalente sobre la atención médica porque, si bien tiene un serio deterioro a causa de la diabetes, dispone de recursos económicos familiares que le posibilitan acudir al Seguro Social o a la medicina privada. Ello no obstante, ninguna instancia le resuelve los múltiples trastornos ocasionados por la diabetes, por lo cual pasa de la satisfacción en ciertos servicios a la insatisfacción en otros.

Una serie de situaciones diferentes ocurre con quienes evalúan la atención médica en términos negativos. La mayoría son mujeres con 
rasgos semejantes: mayores de 60 años, con dos padecimientos en promedio, más años con diabetes (16 en promedio); y quienes presentan gran cantidad de trastornos y efectos negativos en su vida diaria a causa de sus padecimientos. Tampoco cuentan con recursos económicos adicionales, por lo cual consideran el Seguro Social como la única opción a que pueden acudir en forma permanente, recibir medicinas, acudir a especialistas y realizar estudios, sin costos extras para ellos. Casi todos, no sólo terminan con una visión negativa de la atención médica recibida en esta institución, según veremos adelante, sino también con un sentimiento de amargura e impotencia ante la poca o nula mejoría de sus síntomas.

\section{Puntos de vista cambiantes}

Estos sujetos han vivido al menos con un padecimiento crónico durante más de una déca$\mathrm{da}$, en promedio. En consecuencia, su valoración de la atención médica no es estática, sino en función de un continuum en el tiempo, razón por la cual tienen una imagen del presente en comparación con la del pasado. Sobresale aquella en la cual la atención del pasado era cualitativa y cuantitativamente mejor a la del presente, sobre todo en el Seguro Social. Hay quienes han tenido acceso a esta institución desde los sesenta y unos cuantos desde los setenta y los ochenta. Y quienes más tiempo han estado en contacto sostienen que los recursos y el trato han empeorado con los años al argumentar que antes recibían un mejor trato y tenían acceso a más y mejores servicios y recursos, sobre todo medicamentos y suplementos como las vitaminas y el hierro. Miguel Fernández, de 61 años, jubilado y con varias décadas de estar asegurado, se refiere al tema en términos semejantes a como lo describen otros, “... hace unos cuatro, cinco años, daba muy buena medecina, el Seguro (daba) muy buena 'medecina'... usted se aliviaba de una enfermedad según lo quetuviera y daban medecina, vitaminas, iqué sé yo!... y últimamente pos dan puro, la pastilla esa, ¡naproxén!, es lo único que dan...".

Tal cambio, a su vez, es referido por unos cuantos en sentido inverso, ésto es, como parte de una mejoría en la atención recibida con el paso del tiempo. Aquí se trata de dos situaciones diferentes: aquella mejoría por la utilización de un tipo diferente de servicio médico, sobre todo entre quienes acudían al Seguro Social y pasaron a atenderse en la medicina privada o en los servicios públicos asistenciales y, por otra parte, entre quienes pudieron cambiar de médico en la misma institución.

\section{La multiplicidad de servicios empleados}

De acuerdo a las narraciones de casi todos los participantes del estudio, no es posible aludir a una fuente de atención única, porque suelen acudir a varios servicios en forma simultánea para la atención de su padecimiento. Así, su evaluación dista de ser homogénea en tanto quienes acuden a dos o más servicios por lo común catalogan uno en términos positivos y otros de forma diferente. La mayoría de estos sujetos hace referencia a múltiples instancias de atención empleadas al mismo tiempo, y su valoración puede coincidir o diferir en cada caso.

Varias razones se manejan, sin ser excluyentes entre sí, para dar cuenta de tal valoración. Algunos hacen una evaluación doble o triple porque acuden en forma simultánea a más de un servicio médico, sea por no mejorar o haber recibido una atención deficiente; por ejemplo, catalogan la medicina privada en términos positivos, pero sin dejar de asistir a la seguridad social, de la cual tienen una opinión negativa, argumentando que, si dejan de acudir al Seguro, se les podría negar la atención o regañarles en el futuro. Otros, en cambio, los clasifican de esa manera al asistir cíclicamente a ellos; tal es el caso de quienes acuden a la seguridad social y luego a los centros médicos asistenciales en función de los periodos de empleo y desempleo de ellos mismos, sus cónyuges o sus hijos; aunque, en otros casos, se da al acudir a la medicina privada mientras cuentan con recursos económicos y vuelven a la asistencia pública y/o a la seguridad social cuando se les terminan. Esto ocurre entre los enfermos con hijos migrantes en Estados Unidos, al recibir ocasionalmente remesas para pagar los costos de la atención médica.

\section{Sobre los padecimientos}

Tanto los hombres como las mujeres de este estudio distan de evaluar la atención médica sólo a partir de las necesidades y demandas de la diabetes. Su perspectiva también se elabora tomando en consideración los otros padecimientos crónicos que les aquejan. El asunto es relevante porque menos de la mitad sólo padece diabetes, y la mayoría presenta al menos otro padecimiento crónico, aunque no son raros quienes tienen dos o tres adicionales, sobre todo hipertensión y artritis. Muchos, incluso, manifiestan tener más trastornos y de mayor severidad a causa de tales padecimientos que de la misma diabetes, aunque por lo común se da un traslape. Por lo anterior, su valoración de la atención médica abarca el manejo de los 
trastornos de sus padecimientos crónicos y se centra en la institución y profesionales, sobre todo los médicos encargados de los mismos. Los participantes de este estudio valoran en especial aquellas instancias que atienden sus múltiples padecimientos, sea el médico general o el familiar; sea en los servicios públicos, la medicina privada o la seguridad social, y en el centro de salud o el hospital. Pero tal evaluación se dirige a la atención de sus padecimientos, y no de una enfermedade en particular.

\section{Los tipos de servicios}

La mayoría de los participantes toma en consideración múltiples aspectos de los servicios médicos vinculados a la atención de sus padecimientos, sea que los utilice en forma simultánea o sucesiva. Y no obstante sus apreciaciones generales de tales servicios, también suele hacer una evaluación específica de cada uno, del Seguro Social, de los servicios públicos asistenciales y de la medicina privada.

\section{a) El Seguro Social}

Cuatro de los 30 participantes nunca han estado en contacto con el Seguro Social; por ello, aquí aludimos a los comentarios de los 26 individuos que acuden o han acudido al mismo. La opinión general de quienes reciben atención médica en esta institución no puede ser más adversa: sólo dos la consideran buena; unos cuantos tienen una posición ambivalente y la mayoría la califican en términos negativos. La mayoría utiliza frases del tipo "está mal", "es muy malo", "es una anomalía", "allí se enferma uno", "anda muy mal" y "no atienden bien". Un sinnúmero de argumentos sirven para justificar tal conclusión, además de ser considerada la única instancia que ha empeorado con el tiempo. Quienes acuden a esta institución aluden frecuentemente al factor tiempo, pero en términos negativos. En primer lugar, por los largos tiempos de espera para la consulta, los análisis clínicos o estudios de gabinete. Alejandro Becerra, de 35 años y con 2 de vivir con diabetes, expresa su malestar en términos muy semejantes a muchos otros, dado el número de horas dedicadas a la espera de la consulta: “Para empezar a apartar (el lugar), póngale se va a las doce, ll ega usted y pregunta. Ya cuando llega allá a veces hasta dos, tres, cuatro personas adelante, ¿a quéhoras llegarían?, ¿a las once o a las diez dela mañana para alcanzar los primeros Iugares que tienen del horario dela una y media?... el doctor Ilega a las dos o a las tres dela tardea veces, entonces ya empieza a consultar y pos... si le tocó el lugar tres, pos ya va a salir a las tres y media, quince para las cuatro, entonces ya perdió ahí medio día". El factor tiempo también se alude al requerir atención de un especialista o estudios especiales, nada raros entre quienes sufren padecimientos crónicos. Pero el hecho que provoca más desconcierto y molestia es la falta de respeto por el horario de las citas programadas. Como se les cita cada mes o dos meses para su control, al término de la consulta se les programa el día y la hora de la siguiente; ello no obstante, se les exige llegar al inicio del horario de consulta del médico, como si no tuvieran cita programada, y terminan siendo vistos varias horas después; incluso hay quienes manifiestan que, si llegan tarde, se les castiga de varias formas. Emilia Ruiz lo describe así, “... ¡bueno! si nos citaron a las nueve, me voy a las ocho y media, (pero) ino!, tiene que estar ahí a las... cuando mucho a las siete de la mañana... pero nunca nos vamos siquiera a las ocho porqueya, si nos vamos a las ocho, nos de jan hasta las nueve y media, a las diez dela mañana, (porque dicen) ‘¡no, pos ya llegaron tarde!'”. Por último, la alusión al tiempo también aparece en un sentido opuesto, ésto es, porque la mayoría reclama el poco tiempo dedicado por el médico a la consulta.

Estos sujetos con uno o varios padecimientos crónicos han estado en contacto con diversos servicios de las instituciones durante años. Y es a partir de tales contactos que hacen una evaluación específica de cada una de ellas. Así, el mayor número de señalamientos críticos se dirigen a la consulta, a la farmacia, y al laboratorio; en cambio, urgencias y hospitalización son considerados donde se brinda mejor atención, aunque se destacan aspectos negativos como ser demasiado fríos, no tener en cuenta las necesidades de las personas e, incluso, por cierto mal trato. Muy pocos hacen alusión a "Ias pláticas de nutri ción" o a "las sesiones del ejercicio"; pero las opiniones les son favorables en términos de que sirven o son útiles.

Comúnmente se acepta la idea de que el médico juega un papel central en la atención de las enfermedades crónicas. Lo anterior dista de ser verdad, por lo menos entre quienes aquí acuden al Seguro Social. Según se desprende de las narraciones, la atención de su(s) padecimiento(s) crónico(s) implica el contacto con múltiples profesionales; sus comentarios, por ende, hacen referencia a varios de ellos y no siempre en forma homogénea. Pocos califican a los médicos familiares y a las recepcionistas en términos favorables, a diferencia de lo que ocurre con las trabajadoras sociales, nutricionistas y médicos especialistas. Entre los co- 
mentarios relativos a los médicos familiares, destaca que regañan, se enojan, no ayudan, no examinan, no atienden bien, no explican lo necesario o los problemas, todas las molestias las adjudican a la diabetes, no son concientes, su atención es inadecuada, son groseros, no se preocupan por los problemas de sus pacientes, les falta ética, se ponen broncos, no diagnostican correctamente y no prescriben el tratamiento adecuado. Con dificultades para movilizarse por la gravedad de su diabetes y con múltiples complicaciones, a sus 38 años y unos cuantos meses antes de morir, Mercedes Lara nos decía la única ocasión que conversamos sobre el tema: “....ahora que estuve yo en el Seguro me dijeron que meiban a hacer una transfusión... yo no sabía quetenía quellevar un donador y el doctor se portó muy grosero conmigo, o sea, hasta me gritó y ahí se puso muy grosero y él me dijo que la sangre no se regalaba y que tenía quellevar mi donador... si a mí desdeun principio mehubieran dicho que ocupaba donador entonces yo... hubiera buscado quién hubiera ido..."

Otro señalamiento constante remite a la consulta. Haciendo referencia a uno o a varios motivos, los participantes aluden no entender la razón por la cual el diagnóstico del médico familiar difiere del de los médicos privados o del centro de salud; no se les revisa o examina; les prescriben dietas para morirse de hambre, y los médicos se oponen a enviarlos con los especialistas, a pesar de considerar necesitarlos. Cada uno tiene su valoración particular y ofrece ejemplos concretos de las situaciones que les ha tocado vivir. Marina Becerra, de 62 años, con experiencia como enfermera, expresa su insatisfacción por la falta de revisión del médico “...usted va con el doctor y el ya le está haciendo su receta, el doctor si acaso le tomó la presión, jah que bueno!, pero el doctor nunca, nunca se paró de su asi ento para revisarle un pie, una uña, un... pues nada..."

Los comentarios sobre los nutricionistas y trabajadores sociales, en cambio, si bien poco frecuentes, siempre se dan en términos positivos. Varios puntos destacan en sus argumentos: sirven sus pláticas, es de utilidad lo que enseñan y se la pasan bien allí. Ninguno hace referencia a experiencia negativa alguna.

Otro tema recurrente y con una connotación crítica es el de los medicamentos. Un motivo de queja remite a su disponibilidad, es decir, por lo general se les indican en gran cantidad, pero no se encuentran en la farmacia, por lo cual deben regresar una o varias ocasiones a la clínica o volver con el médico a que les indique un sustituto. Otro es la apreciación sobre la eficacia de los medicamentos, al considerar que están "rebajados", al no servir para el control ni lograr una mejoría; producen efectos secundarios, y siempre se prescriben los mismos, a pesar de su ineficacia y efectos indeseables. En este contexto, si bien la farmacia cuenta, por lo general, con medicamentos para la diabetes, el problema crítico lo consideran por la falta de aquellos a los cuales asignan igual o mayor importancia, como son para la artritis y la hipertensión. Además, casi todos han escuchado o percibido que los medicamentos adquiridos en la farmacia comercial ofrece mejores resultados que los de la institución debido a que están "rebajados". Estos motivos generan malestar, tal como se desprende del comentario de Mercedes Lara, “... en el Seguro la mayoría de la medicina no la hay... una vez me dieron una receta que duré quince días, 'y que pasado mañana, y quetal día', y mandaba y mandaba, por fin un día ya no mandé, al cabo ya no mevan a dar nada. Ya me dijo el doctor otro día que fui ¿quéle dieron?, me recetaron unas pastillas y unas vitaminas, pero pues ni melas dieron, y él vio la lista y dijo: ‘ni levoy a dar, porque todavía no hay"'.

Todo lo anterior se traduce, a juicio de estos sujetos, en una insatisfacción por la atención brindada en esta institución y en los resultados: no sienten mejoría, no controlan su azúcar en sangre, e inclusive con cierta frecuencia se sienten mal por los efectos secundarios de los medicamentos. Rubén Romo, jubilado y a sus 64 años, es uno de los pocos convencidos de la buena atención en el Seguro Social. Sin embargo, no deja de reconocer los efectos provocados por los medicamentos, cuando señala: “Bueno, la medicina sí me cura, pero por decirle, meirrita mi estómago, meirrita mis riñones, me irrita otra cosa, me cura una cosa, pero me perjudica otra..."

\section{b) Los servicios públicos asistenciales}

Mesa-Lago (1992) reporta que el gasto per cápita del Seguro Social en México, en 1992, fue casi el doble del de la Secretaría de Salud. Desde la perspectiva de la población enferma, sin embargo, tales diferencias distan de expresarse en forma mecánica en la atención que reciben. Trece participantes han acudido a la asistencia pública (sea centro de salud u hospital) para la atención de su(s) padecimiento(s) crónico(s). Con la excepción de una mujer que la compara con la recibida en Estados Unidos, Ios restantes la catalogan unánimemente como buena. En torno a una serie de expresiones del tipo "reci bo ayuda" o "mees de utilidad", se encuentra un conjunto de señalamientos semejantes 
sobre este tipo de servicio. Se trata de una vaIoración que abarca poco tiempo, es más reciente y de menor continuidad respecto a la de quienes acuden a la seguridad social. Sólo una mujer entrevistada alude a la atención recibida por un periodo largo de tiempo, 16 años, el resto se refiere al servicio otorgado al momento de las entrevistas y/ o durante los meses o años inmediatos anteriores.

Esta apreciación conlleva una disociación entre los distintos niveles y áreas de los servicios. Entre otra, los centros de salud son considerados como entidades ajenas a los hospitales de la misma Secretaría de Salud; de hecho quienes acuden a estos hospitales lo hacen en forma directa, sin ser referidos, o sólo en forma ocasional por los médicos de los centros de salud. Lo mismo ocurre con las distintas unidades las cuales suelen encontrarse en lugares diferentes; por ejemplo, el centro de salud es visto como unidad de consulta, el de análisis clínicos como tal, y el de hospitalización como una entidad ajena a todo lo anterior. El comentario de Juana Cárdenas es significativo al respecto "estuve pagando el Seguro por unos cuatro años, deahí me pasaron al Hospital (Civil), ahí estuve en control como cinco años y me enfadé de ir... y pues ya, pos otra vez al centro desalud ..."

Los participantes de este estudio se refieren al quehacer del médico en lo fundamental, aunque la enfermera y la trabajadora social aparecen de forma marginal. A excepción de dos sujetos, todos catal ogan a los médicos en términos favorables: son amables, se interesan por sus problemas, los consuelan, los felicitan, los aconsejan, les platican, por lo general no les prescriben insulina, están capacitados y les brindan buena atención. Rosario Cárdenas, al igual que otros, se refiere al médico del centro en términos positivos “... pos sí me ha servido mucho y como ledigo me ayudó mucho cuando yo me sentí mal, me dio consuelo, o sabe, será porqueellos son doctores, me imagino que es como un padre quele aconseja a uno ¿verdá?, no sési será por cada persona... y yo con ese doctor para mí mesirvió mucho; ahora, cada vez que voy, dice que me fel icita porque me he cuidado, me ha control ado...". Esta apreciación favorable se acentúa porque en ocasiones les proporcionan medicamentos e, incluso, al enviárselos a su casa, darles más libertad de comer y no prescribirles insulina.

Los tiempos de espera, a su vez, son considerados más satisfactorios por ser más cortos al asistir a consulta, al acudir con otro especialista y al respetarse el horario de las citas. Micaela González, al igual que otro(a)s, a pesar de ser derechohabiente del Seguro Social y residir a sólo cinco cuadras de su clínica de adscripción, prefiere acudir a un hospital público asistencial; el mismo se encuentra a una hora de distancia, y ella debe tomar dos vehículos de transporte público. A ello se refiere cuando nos platicaba una tarde "son diez pesos lo que me cobran de consulta (en el hospital público), no, idiez no!, trece o catorce, son lo que cobran de consulta y allí es rápido, allí es citas, no es que 'espérese', allí va uno y le dan su cita 'vienea tales horas', ... supongamos quetengo mi cita a las cinco, me voy a las tres y media, ll ego allá a las cuatro, cuatro y media y ya Il ego, 'vengo con el doctor fulano', 'sí, si éntese', para pronto, luego luego me meten, no duro ni la hora ahí, no duro ...". Es paradójica esta situación, siente más satisfacción por un proceso que le lleva cuatro horas y media y en el cual requiere tomar cuatro vehículos públicos, respecto a las mismas horas que permaneció en su clínica, ubicada a cinco cuadras de su casa, en la última consulta con el médico familiar del Seguro Social.

No obstante lo anterior, una situación contradictoria es señalada por unos cuantos respecto a los medicamentos y a los estudios clínicos. Reconocen agradecidos los esfuerzos de los médicos por proporcionarles los medicamentos, entre ellos muestras médicas, así como sus sugerencias para encontrar el laboratorio más económico o realizar los estudios necesarios, cuando no lo hacen en la misma institución. Pero, al mismo tiempo, argumentan serias dificultades para la compra de los medicamentos o la realización de los estudios. Lo primero es valorado en términos positivos, pero lo segundo terminan considerándolo del ámbito de competencia personal y/o familiar, mas no de los médicos, o de la institución de salud. Tales acciones son consideradas como un favor otorgado por los médicos.

\section{c) La medicina privada}

Más de la mitad de los participantes opinan sobre la medicina privada por el contacto directo que han tenido con ella en el manejo de su(s) padecimiento(s) crónico(s). Muy pocos acuden a estos servicios como fuente permanente de atención y en forma intermitente, los más ocurren en forma esporádica y sólo en momentos de crisis y/o al contar con recursos para sufragarla, pero todos coinciden en valorarla en términos positivos o en forma ambivalente. Son comunes al respecto expresiones del tipo "estoy muy agusto", “bien, muy sati sfecha” o "me atienden muy bien". Tales comentarios aluden fundamentalmente a la atención recibida del médico general asentado en el mismo barrio, 
pero en tres áreas consi deradas ajenas entre sí: el consultorio, el laboratorio de análisis clínicos y la farmacia. En este contexto, se excluye cualquier alusión a la hospitalización o a otro servicio de apoyo.

El médico es aquí la figura central. Incluso los responsables de los análisis y encargados de la farmacia se pierden en una serie de alusiones al laboratorio y a la farmacia. Un sinnúmero de comentarios favorables se hace de los médicos: les hablan por su nombre, conocen sus problemas, les dan palabras de aliento, los examinan, les platican, se les puede hablar por teléfono, hay quien los visita en su casa cuando están enfermos, no los citan a consulta mientras se sienten bien, cambian los medicamentos cuando no funcionan, no les prescriben insulina o la retiran cuando otro médico la ha indicado, les prescriben vitaminas, son más tolerantes con la dieta, aceptan el consumo de refrescos o carne en cantidad limitada, son buenos médicos y están bien capacitados.

Los análisis clínicos y los medicamentos también son valorados en términos positivos. Los primeros, al considerar que sus resultados son más confiables que los de las instituciones públicas, por el menor número de estudios realizados, al ser menor el riesgo de confusión entre sí y mayor el cuidado en su elaboración, además de ajustarse a los tiempos de las personas. Alejandro Becerra, de 35 años y dos de padecer diabetes, nos explicaba un día las razones por las cuales va a consulta con el médico del Seguro Social, y se hace los análisis en un laboratorio privado “...los análisis del Seguro si empre sale uno arriba, si empre, nunca sale uno normal... yo siempre me los hago en un laboratorio clínico (privado)... y me lo hago ahí y melos hago en el Seguro y siempresalgo aquí en Ios laboratorios normal...". Los medicamentos, a su vez, también son considerados de mejor calidad respecto a los de las instituciones de salud, al percibir en forma notoria sus efectos benéficos, al disminuir las molestias, los niveles de azúcar, y sentir menos efectos secundarios.

Casi todos reconocen las "limitantes" de este tipo de medicina. Se trata del costo elevado de la consulta, de los estudios clínicos y de los medicamentos. Según casi todos, ello los obligan a utilizarlos sólo en forma periódica o durante las crisis. Micaela González nos decía un día al respecto, “... (el médico) te dedica más ti empo... (pero) cada ocho días son 150 pesos, más la medicina, si ya se meterminó esa, la me dicina mecuesta 300 y 300 (pesos), jno crea!..."

A pesar de sus el evados costos, una de las convicciones más generalizadas entre los participantes es que este tipo de servicio es el me- jor en cuanto al tiempo de espera. Esto no significa que siempre reciban la atención en forma inmediata; algunos reconocen, inclusive, el hecho de que numerosas personas pueden solicitar la misma atención médica en el mismo momento. Tal asunto, sin embargo, no se le vaIora en términos negativos por dos razones: porque a todos se les da una ficha y cada quien decide si espera o regresa a una hora aproximada, en tanto casi todos los consultorios están en el mismo barrio; por otra parte, donde hay muchas personas solicitando consulta con el mismo médico suele ser considerado en términos positivos porque constituye la prueba irrefutable de que se trata de un buen médico.

El resultado final es una opinión en donde no sólo manifiestan sentirse más controlado(a)s del azúcar y con menos molestias, sino que incluso enfatizan su confianza en el médico. Por ello, varios participantes están dispuestos a hacer sacrificios, sobre todo económicos, para seguir atendiéndose con ellos; otro(a)s, incluso, se preocupan y angustian cuando el médico se enferma o cuando cambia su consultorio a otra zona de la ciudad. Eloísa M éndez manifiesta tal preocupación en los siguientes términos, “...(cuando) estoy mala y que es domingo, pues me aguanto hasta que llegue él, porque yo no quiero otro doctor... el otro día dijeron que estaba malo y que le había dado hasta embolia... fíjese que yo me preocupétanto, dije ¿qué voy a hacer yo?, yo nomás pensaba en mí, no en tanto paciente queviene desde sabe dondecon él..."

\section{Discusión y comentarios finales}

Varios objetivos nos propusimos en este trabajo: dar cuenta de la perspectiva de sujetos de la clase trabajadora de un barrio urbano popular sobre la atención médica relativa a su padecimiento crónico; valorar si existen diferencias en tal perspectiva en función del tipo de servicios médicos y explicar su punto de vista teniendo presente el contexto material y social en que se encuentran inmersos.

Nuestros resultados difieren de los hallazgos de otros autores quienes reportan un porcentaje elevado de usuarios satisfechos o con una opinión favorable de los servicios de salud. Ramírez-Sánchez et al. (1998) y Ruiz et al. (1990) encuentran que el $81,2 \%$ y el $97 \%$ de su población estudiada los consideran buenos o excelentes; en cambio, nosotros encontramos una situación casi opuesta porque más de la mitad de los participantes los catal ogan como ambivalentes o malos-deficientes. Tales diferencias podrían ser explicadas por varios factores. En 
primer lugar se trata de poblaciones distintas. Los primeros corresponden a los usuarios de una Encuesta Nacional de Salud que acudieron por diversos motivos a un servicio de salud durante las dos últimas semanas; los segundos son pacientes o familiares que asistían a un hospital del tercer nivel. También hay diferencias metodológicas; la información de ambos se obtuvo mediante una encuesta, en el primer caso aplicada en el hogar y en el segundo en el hospital. Y si bien nuestro estudio coincide parcialmente con aquellos datos que consideran buena la atención recibida en los servicios públicos, incluida la atención hospitalaria, debe tenerse presente que la administración de cuestionarios el eva significativamente el porcentaje de respuestas satisfactorias respecto a otras técnicas empleadas (Lewis, 1994).

En términos semejantes a otros estudios, como el de la Fundación Mexicana para la Salud (FMS, 1994), sobre la satisfacción de los usuarios de los servicios de salud, nuestros hallazgos indican que más de la mitad de los sujetos con un padecimiento crónico no catalogan la atención médica recibida como buena o satisfactoria. Ambos resultados nos parecen de mayor confiabilidad porque los participantes tuvieron gran libertad al dar su opinión sobre el tema en su hogar, los datos se obtuvieron durante varias entrevistas y como parte de la narración de sus experiencias con su padecimiento, razones por las cuales asumimos que hubo mayor confianza para exponer su punto de vista sobre tales servicios. El aporte fundamental del trabajo aquí realizado no consiste en las cifras encontradas, ni por la posibilidad de utilizar estos resultados para "tener evidencias" de la mala calidad de los servicios públicos y, por ende, proponer su privatización. Ni los objetivos del estudio, ni la metodología empleada, permiten Ilegar a tales conclusiones. Su aporte, más bien, deriva de las problemáticas que emergen del mismo en relación a la experiencia de sujetos enfermos en la búsqueda de atención a su padecimiento.

En el marco de nuestros resultados, no asumimos que cada enfermo construye su propia realidad sanitaria en una suerte de postura interpretativista a ultranza. Según los datos expuestos, ciertos elementos del contexto y del orden físico parecen explicar la percepción e interpretaciones entre quienes tienen un punto de vista bueno o favorable de la atención médica porque no sólo tienen una trayectoria estable, sino también disponen de recursos que les posibilitan tener más opciones para utilizar distintos tipos de servicios, sea la medicina privada y/ o los servicios públicos. En cambio, una combinatoria fatídica se dá entre quienes tienen una trayectoria deteriorante y pocas o nulas opciones de atención, lo cual les obliga a acudir ineludiblemente a la seguridad social y/ o a los servicios públicos asistenciales. La perspectiva de los primeros es favorable porque tienen múltiples posibilidades de atención y pocas manifestaciones o efectos secundarios del padecimiento; los segundos se ven obligados a emplear la única alternativa médica existente y con múltiples síntomas y/ o complicaciones. Una hipótesis a comprobar aquí es si quienes perciben e interpretan tales servicios como deficientes no cuentan con recursos para atenderse en la medicina privada y no acuden a los servicios públicos dado el creciente número de trastornos que les obliga a acudir al Seguro Social por la diversidad de servicios que les ofrece. Quienes consideran la atención médica mala o deficiente, en este contexto, son hombres y mujeres ancianos, con más padecimientos, con mayores necesidades de atención y con menores recursos económicos. Su única opción parcial parece ser la posibilidad de cambiar de médico y/o de asistente.

Este estudio también ilustra las dificultades y limitantes que enfrentan los trabajos sobre la satisfacción de los usuarios o sobre la calidad de los servicios médicos (Garrocho, 1995). De acuerdo a las circunstancias descritas para estos sujetos del sector popular con padecimiento(s) crónico(s), resulta poco probable contar con una valoración única y definitiva de los servicios de salud en tanto su acceso a los mismos cambia a lo largo del tiempo, al acudir a varias instituciones a la vez. En el marco de las condiciones generales de empleo de la sociedad en su conjunto, sectores importantes de la población tienen acceso a la seguridad social durante cortos periodos de tiempo, para luego perderlo a causa de su expulsión al mundo del desempleo. Una limitante adicional deriva del desconocimiento que se tiene del significado que le dan los sujetos a conceptos tales como calidad o satisfacción, máximo cuando sus referencias se circunscriben a sus experiencias o las de su red social cercana con los servicios de salud. En este marco, resulta insostenible generalizar los resultados sobre la satisfacción de cierta población a partir de los datos de otra: por ejemplo, cuando se aplican los de un país anglosajón a los sectores medios o populares de los países lati noamericanos (Gattinara et al., 1995). Otra problemática remite al conjunto de elementos subjetivos que se emplean y combinan para definir determinado tipo de perspectiva; los largos tiempos de espera en la consulta, por poner un caso, dicen poco por sí mis- 
mos; sin embargo, pueden tener significados completamente diferentes al visualizarlo en la medicina privada o en el Seguro Social. Lo mismo parece suceder con los medicamentos y la indicación de medidas terapéuticas, como en el caso de la dieta.

¿De cuál perspectiva estamos hablando, después de todo? Para el caso que nos ocupa, al tener presente a enfermos crónicos que requieren servicios médicos a lo largo del tiempo, la pregunta dista de ser obvia. Quienes padecen diabetes y otras enfermedades crónicas no hacen un corte y se aíslan en el tiempo, en tanto viven y asumen la atención recibida como un fenómeno cambiante. Quienes manejan su(s) padecimiento(s) en determinados servicios médicos durante más de una década destacan el deterioro creciente de la atención médica, sobre todo del Seguro Social. Este dato pudiera corresponder, a su vez, a indicadores macroeconómicos donde se muestra, entre otras cosas, una inversión cada vez menor en tal institución (Mesa-Lago, 1992).

Pero la pregunta también remite a la aparente contradicción de la doble o triple evaluación, con frecuencia disímbola, que hacen estos sujetos del sector popular sobre los servicios médicos. Tal situación puede ser explicable según se ha mostrado desde los trabajos pioneros de Mechanic (1962) y Suchman (1992), porque los sujetos enfermos usan distintos servicios de salud en su "carrera de enfermo". Dejando de lado la utilización de otros modelos de atención a la enfermedad "no médicos", aquí constatamos que estos enfermos crónicos evalúan y comparan en forma permanente los distintos tipos de atención. Así, mientras califican la atención de cierta institución como buena, al mismo tiempo pueden estar catalogando otra como mala o pésima en función de su experiencia concreta con ellas. Esta valoración múltiple da cuenta de la compleja realidad que enfrentan los miembros del sector popular al verse forzados a cambiar de atención médica a causa del círculo empleo-desempleo al cual entran o salen ellos o sus hijos, o ante la existencia de recursos económicos adicionales durante cierto tiempo, lo cual les permite acudir a los médicos privados para luego volver a los servicios públicos. El mismo fenómeno ocurre con su apreciación de la institución con la cual están en contacto; frente a los servicios de consulta externa, farmacia y laboratorio del Seguro Social, también están en contacto con los de urgencias, hospitalización y trabajo social o de Ios nutricionistas. Los primeros suelen ser catalogados en términos negativos y los segundos como buenos o ambivalentes.
La evaluación de la atención médica en torno a la diabetes, por otra parte, dista de dejar de lado las circunstancias de otros padecimientos crónicos. Por lo común tal apreciación se hace en función del problema considerado de mayor gravedad, tal sería el caso de una hiper o hipoglucemia, pero se puede dar igual o mayor peso a la atención requerida para los síntomas de la hipertensión y/ o la artritis. Desde la perspectiva de quien sufre, se trata de la experiencia del padecimiento, por lo cual la atención recibida es vista como una unidad (Conrad, 1987). Así, poco o nulo interés tienen en precisar si tal o cual manifestación es expresión de alguna enfermedad en particular, cuando su preocupación es limitar al máximo sus efectos o lograr desaparecerlos. En este sentido, un médico o un servicio es valorado en función de su capacidad de eliminar o controlar el conjunto de molestias, más que la forma como las conciben o las tratan.

Según Zurita et al (1997), 44\% de los mexicanos opinan que los mejores servicios de consulta general son de la medicina privada, para otro $26 \%$ del Seguro Social y para el $15 \%$ restante de la Secretaría de Salud. Según estos autores, tales hallazgos son representativos de todos los adultos del país en 1994. Nuestros hallazgos, haciendo referencia a enfermos de un barrio urbano, también evidencian una percepción diferencial de los distintos tipos de servicios, pero difieren en varios sentidos. Por un lado, no existe correspondencia entre los recursos de los servicios frente a la perspectiva de la población por la atención recibida, por los menos en lo relativo al Seguro Social y a la Secretaría de Salud. Esta apreciación dista de ser equivalente al ser valoradas múltiples dimensiones, recursos e instancias del primero, mientras que la segunda es vista en forma parcial, sobre todo a través de la consulta externa y el quehacer de los médicos. Ello no significa dejar de reconocer la valoración tan diferente en aspectos específicos, como es el trato de los médicos en la consulta. Los médicos de la seguridad social son catalogados como sujetos fríos, poco interesados en los problemas de los enfermos, autoritarios y poco dados a escuchar, palpar o revisar; lo inverso parece ocurrir con los médicos asistenciales y de la medicina privada. No obstante tales hallazgos, ciertas evidencias sugieren que tales puntos de vista tienen distintos significados; por poner un caso, los derechohabientes del Seguro Social critican la atención recibida y demandan mejores servicios argumentando su derecho por pagar parte del mismo; en cambio, quienes acuden a los servicios públicos suelen considerarlos en 
términos muy distintos y como un favor otorgado, básicamente por el médico. En ningún momento hacen consideración alguna sobre su derecho a la salud.

Varias implicaciones derivan de los hallazgos de este trabajo. El mismo se centra en la perspectiva de sujetos de un barrio popular urbano sobre la atención médica en torno a su(s) padecimiento(s) crónico(s). Tal perspectiva ni es la única ni la verdadera; de las múltiples existentes, cada una tiene su sustento, ofrece sus resultados y sus sesgos. Los profesionales de la salud adoptan una perspectiva "científica", pero se trata de una visión externa y ajena al individuo que padece. La "comunidad" o determinados grupos sociales tienen una visión más general pero inespecífica del fenómeno, la que, con frecuencia, incorpora la de quienes nunca han requerido atención médica, pero cuya opinión puede pesar en los servicios o en el ámbito público; aquí el riesgo es dar cuenta de las representaciones más no de las experiencias. Otra óptica es la de los usuarios de los servicios; se caracteriza por incluir sólo a quienes acuden a ellos pero deja de lado la de quienes utilizan raramente esa instancia de atención, o no la utilizan. Otra es la de los cuidadores, sean integrantes del grupo doméstico o de la red social, encargados de apoyarlos, y utilicen o no los servicios médicos. Esta puede ofrecer una visión complementaria sobre las necesidades, las prácticas y las experiencias de los sujetos enfermos que pueden pasar desapercibidas. La perspectiva de los sujetos enfermos, en este contexto, se sustenta precisamente en la experiencia de quien vive día a día con el padecimiento, aún cuando también da cuenta de sus

\section{Agradecimientos}

La participación de los sujetos enfermos ha sido decisiva en el estudio; nuestro agradecimiento a todos ellos. También a los doctores Leticia Robles, René Leyva, Bernardo Jiménez y Andrew Roth por sus valiosos y estimulantes comentarios. Dos evaluadores(as) anónimos(as) también nos han brindado comentarios de suma pertinencia.

Este trabajo contó con el apoyo financiero del Consejo Nacional de Ciência y Tecnologia del Gobierno de M éxico, acuerdo 4246P-M 9608. representaciones y prácticas. Así, no sólo es más específica sino que incluye a quien acude a las distintas instancias de atención, sean médicas o no, y puede dar cuenta de la multiplicidad de aspectos ligados a su vida diaria. Pero la perspectiva de los sujetos enfermos con el mismo padecimiento parece no ser homogénea; quedaría por indagar si existen variaciones, por ejemplo, en función de su pertenencia a distintas clases sociales.

Nuestros hallazgos se vinculan a la situación actual y a las reformas propuestas para transformar el sector salud; una de ellas remite a la privatización de los servicios públicos, incluida la seguridad social. Diversos sectores sociales se han manifestado a su favor, otros en contra; pero nos parece que sectores de la misma población, incluso del popular como aquí hemos mostrado, poco dispuestos estarían en apoyar su defensa, cuando hacen una valoración negativa de ella y buscan la posibilidad de contar con una atención más digna y eficiente para enfrentar sus padecimientos (crónicos). Pero esta valoración crítica no se aplica a todos los servicios públicos, según se desprende de los datos expuestos; los participantes también tienen una valoración favorable de los servicios públicos asistenciales. Pero defender a ultranza las bondades de los servicios públicos implica negar estos y otros resultados semejantes que hacen referencia a una gran insatisfacción ante ciertos servicios médicos. Dada la relevancia social y política del asunto, consideramos urgente la necesidad de continuar esta línea con estudios que comparen distintos sectores de la población así como con diseños y metodologías variadas.

\section{Referencias}

AGUIRRE, H., 1990. Evaluación de la calidad de la atención médica. Expectativas de los pacientes y de los trabajadores de las unidades médicas. Salud Pública de México, 32:170-180.

BULLER, M. \& BULLER, D., 1987. Physicians' communication style and patient satisfaction. Journal of Health and Social Behavior, 28:375-388.

CASTRO, R., 1995. The subjetive experience of health and illness in Ocuituco: A case study. Social Science and Medicine, 41:1005-1021.

CONRAD, P., 1987. The experience of illness: Recent and new directions. In: The Experience and Management of Chronic IIIness (J. Roth \& P. Conrad, eds.), pp. 1-31, Greenwich: Jai Press Inc.

FMS (Fundación Mexicana para la Salud), 1994. Economía y Salud. Propuestas para el Avance del Sistema de Salud en México. Visión de Conjunto. México, D.F.: FMS. 
GARROCHO, C., 1995. Análisis Socioespacial de los Servicios de Salud. Accesibilidad, Utilización y Calidad. Toluca: El Colegio Mexiquense/ DIF.

GATTINARA, B.; IBACACHE, J.; PUENTE, C.; GIACONI, J. \& CAPRATA, A., 1995. Percepción de la comunidad acerca de la calidad de los servicios de salud públicos en los distritos Norte e Ichilo, Bolivia. Cadernos de Saúde Pública, 11:425-438.

GONZALEZ-DE-LA-ROCHA, M., 1986. Los Recursos de la Pobreza. Familias de Bajos Ingresos de Guadalajara. Guadalajara: El Colegio de Jalisco/ CIESAS/SPP.

GUMPERZ, J., 1995. Interviewing in intercultural situations. In: Talk at Work. Interaction in Institutional Settings (P. Drew \& J. Heritage, eds.), pp. 302-327, Cambridge: Cambridge University Press.

HERNANDEZ, P.; ZURITA, B.; RAMIREZ, R.; ALVAREZ,

F. \& CRUZ, C., 1997. Las cuentas nacionales de salud. In: Observatorio de la Salud. Necesidades, Servicios, Políticas (J. Frenk, ed.), pp. 119-142, México, D.F.: Fundación Mexicana para la Salud.

HERZLICH, C. \& PIERRET, J., 1988. De Ayer a Hoy: Construcción social del enfermo. Cuadernos Médico Sociales, 43:21-30.

HOPTON, J.; HOWIE, J. \& PORTER, M., 1993. The need for another look at the patient in general practice satisfaction surveys. Family Practice, 19:82-87.

HUBY, G., 1997. Interpreting silence, documenting experience: An anthropological approach to the study of health service users' experience with HIV/ AIDS care in Lothian, Scotland. Social Science and Medicine, 44:1149-1160.

JUNG, H.; VAN-HORNE, F.; WENSING, M.; HEARNSHAW, H. \& GROL, R., 1998. Which aspects of general practitioners behaviour determine patients evaluation of care? Social Science and Medicine, 47:1077-1087.

LARA, F. N.; TAMEZ, G. S. \& EIBENSCHUTZ, H. C., 2000. Investigación de necesidades y utilización de servicios de salud. Un campo en consolidación. In: Análisis Cualitativo en Salud: Teoría, Método y Práctica (F. J. Mercado \& T. L. Torres, comps.), pp. 81-96, Guadalajara: Plaza y Valdez/ Universidad de Guadalajara.

LAURELL, A. C., 1991. Crisis, neoliberal health policy, and political processes in Mexico. International Journal of Health Services, 21:457-470.

LEWIS, L., 1994. Patients views on quality care in general practice: Literature review. Social Scienceand Medicine, 39:655-670.

LIKE, R. \& ZYZANSKI, S., 1987. Patient satisfaction with the clinical encounter: Social psychological determinant. Social Science and Medicine, 24:352-357.

LINDER-PELZ, S., 1982. Toward a theory of patient satisfaction. Social Science and Medicine, 16:577-586.

MERCADO, F. J., 1996. Entre el Infierno y la Gloria: La Experiencia dela Diabetes en un Barrio Urbano deGuadalajara. Guadalajara: Universidad de Guadalajara.

MERCADO, F. J., 1998. El manejo del tratamiento médico en el marco de la vida diaria. La perspectiva de los enfermos con diabetes. Relaciones, 74:79-93.

MERCADO, F. J.; ROBLES, S. L.; RAMOS, H. I.; MORENO, L. N. \& ALCANTARA, H. E., 1999. La perspectiva de los sujetos enfermos. Reflexiones sobre el pasado, presente y futuro de la experiencia del padecimiento crónico. Cadernos deSaúdePública, 15:179-186.

MERCADO, F. J.; ROBLES, S. L.; RAMOS, H. I.; TORRES, T.; MORENO, L. N. \& ALCANTARA, H. E., 1998. La trayectoria de los enfermos con diabetes en el sector popular de Guadalajara. In: Investigación Cualitativa en Salud. Perspectivas desde el Occidente de México (F. J. Mercado \& L. Robles Silva, comps.), pp. 223-246, Guadalajara: Universidad de Guadalajara.

MERCADO, F. J. \& TORRES L. T., 2000. Análisis Cualitativo en Salud; Teoría, Método y Práctica. M éxico: Plaza y Valdez/Universidad de Guadalajara.

MERCADO, F. J., VILLASEÑOR, M. \& LIZARDI, A., 1999. Situación actual de la investigación cualitativa en salud. Un campo en consolidación. Revista Universidad deGuadalajara, 17:19-30.

MESA-LAGO, C., 1992. Health Care for the Poor in Latin America and the Caribbean. Arlington: Inter-American Foundation.

MINAYO, M. C., 1997. El Desafío del Conocimiento. Investigación Cualitativa en Salud. Buenos Aires: Lugar Editorial.

MODENA, M., 1990. Madres, Médicos y Curanderos: Diferencia Cultural eldentidad Ideológica. México, D.F.: Ediciones de la Casa Chata.

PINHEIRO, R. S. \& TRAVASSOS, C., 1999. Estudo da desigualdade na utilização de serviços de saúde por idosos em três regiões da cidade do Rio de Janeiro. Cadernos de Saúde Pública, 15:487-496.

PODER EJECUTIVO FEDERAL, 1995. Programa de Re forma del Sector Salud. 1995-2000. México, D.F.: Poder Ejecutivo Federal.

RAMIREZ-SANCHEZ, T.; NAJERA-AGUILAR, P. \& NIGENDA-LOPEZ, G., 1998. Percepción de la calidad de la atención de los servicios de salud en México: Perspectiva de los usuarios. Salud Pública de México, 40:3-12.

ROCHA, J. P.; BORGES, J. B.; INACIO, K. L. \& BARCELLA, R. C., 1997. Enfermagem, um serviço de qualidade? O paciente responde. Revista Brasileira da Enfermagem, 50:153-162

RODRIGUEZ, G.; GIL, J. \& GARCIA, E., 1996. Metodología de la Investigación Cualitativa. Granada: ALJIBE.

RUIZ, G. C.; VARGAS, V. F.; CASTILLO, R. C.; PEREZ, P. L. \& MARTINEZ, M. R., 1990. Lo que opinan los pacientes del Instituto Nacional de la Nutrición Salvador Zubirán (INNSZ). Revista de Investigación Clínica, 42:298-311.

SANTOS, M. P., 1995. Avaliação da qualidade dos serviços públicos de atenção à saúde da criança sob a ótica do usuário. Revista Brasileira da Enfermagem, 48:109-119.

SELBY, H. A.; MURPHY, A. D. \& LORENZEN, S. A., 1990. The Mexican Urban Household; Organizing for Self-Defense. Austin: University of Texas Press.

WEBER, R., 1990. Basic Content Analysis. Newbury Park: Sage Publications.

WHO (World Health Organization), 1990. Measuring Consumer Satisfaction with Care. Copenhagen: WHO.

ZURITA, B.; NIGENDA, G. \& RAMIREZ, T., 1997. Encuesta de satisfacción con los servicios de salud. In: Observatorio de la Salud: Necesidades, Servicios, Políticas (J. Frenk, ed.), pp. 237-252, México, D.F.: Fundación Mexicana para la Salud. 